\title{
Synthesis of Optimal Control and Flight Testing of an Autonomous Circular Parachute
}

\author{
Oleg A. Yakimenko, ${ }^{*}$ Vladimir N. Dobrokhodov, ${ }^{\dagger}$ and Isaac I. Kaminer ${ }^{\ddagger}$ \\ Naval Postgraduate School, Monterey, California 93943 \\ and \\ Scott H. Dellicker ${ }^{\S}$ \\ U.S. Army Yuma Proving Ground, Yuma, Arizona 85365
}

\begin{abstract}
Development of guidance and control algorithms for autonomous solid circular parachutes is addressed. This effort is a part of the Affordable Guided Airdrop System that integrates a low-cost guidance and control system into fielded cargo air delivery systems. First, the underlying Affordable Guided Airdrop System concept, architecture, and components are described. Then a synthesis of a classical optimal control based on Pontrjagin's maximum principle is suggested. Then the development of a practical control algorithm is detailed. Simulation and flight-test results of the final Affordable Guided Airdrop System demonstration are also presented.
\end{abstract}

\section{Introduction}

C URRENTLY, high-altitude/low-opening and high-altitude/ high-opening airdropped personnel are the only assets that can be released from altitudes above $1500 \mathrm{~m}$ while still realizing an acceptable landing accuracy. Aerial missions over Bosnia in 1993 underscored high-altitude airdropped payload delivery accuracy concerns during operations conducted from above $3000 \mathrm{~m}$ for resupply and humanitarian purposes. (A lot of cargo ended up at the wrong spots.) Humanitarian-relief airdrops over Kosovo in 1999 and Afganistan in 2001 demanded that airdrop aircraft operate from even higher altitudes, with an expected further degradation of payload delivery accuracy.

This urgent need to improve the point-of-use delivery, that is, "getting the materiel where it needs to be and when it needs to be there," has led to the Affordable Guided Airdrop System (AGAS) project, initiated by the U.S. Army and the U.S. Air Force in late 1990 s. ${ }^{2,3}$

The main goal of the AGAS development is to provide a precision airdrop capability for a delivery system consisting of an existing fielded cargo parachute system such as G-12 and a standard delivery container A-22. From the cost standpoint, it is much more effective to use thousands of flat circular parachutes available in the inventory augmented with a cheap and reliable guidance, navigation, and control (GNC) unit rather than to design and manufacture new more sophisticated and much more expensive high-performance aerodynamic decelerator systems.

The key ideas of the AGAS concept, developed in Refs. 3 and 4 , are discussed next. The first step is for the end user to broadcast a supply request that includes information on where and when it is needed on the ground. On arrival in the vicinity of the assigned

Presented as Paper 2002-4753 at the AIAA Guidance, Navigation, and Control Conference, Monterey, CA, 6-9 August 2002; received 6 December 2002; revision received 28 April 2003; accepted for publication 29 April 2003. This material is declared a work of the U.S. Government and is not subject to copyright protection in the United States. Copies of this paper may be made for personal or internal use, on condition that the copier pay the $\$ 10.00$ per-copy fee to the Copyright Clearance Center, Inc., 222 Rosewood Drive, Danvers, MA 01923; include the code 0731-5090/04 \$10.00 in correspondence with the CCC.

*Research Associate Professor, Dept. of Aeronautics and Astronautics; oayakime@nps.navy.mil. Associate Fellow AIAA.

${ }^{\dagger}$ National Research Council Research Associate, Dept. of Aeronautics and Astronautics; vldobr@nps.navy.mil. Member AIAA.

¥Associate Professor, Dept. of Aeronautics and Astronautics; kaminer@ nps.navy.mil. Senior Member AIAA.

${ }^{\S}$ Director, Air Combat and Soldier Systems; Scott.Dellicker@yuma. army.mil. Senior Member AIAA. drop zone (DZ), a support aircraft (possibly an unmanned air vehicle) drops a wind dropsonde. The wind profile acquired during this drop allows computation of the reference trajectory (RT) and of the computed air release point (CARP). (As a part of the AGAS effort, the Charles Stark Draper Laboratory and Planning Systems, Inc., developed a highly sophisticated system that provides accurate prognosis of the wind over the DZ and generates a CARP and $\mathrm{RT}^{5}{ }^{5}$ ) The delivery aircraft will then be navigated to that point for air delivery of the materiel (payload). Should the wind estimate and calculation of the CARP be perfect, and should the aircrew get the aircraft to this point precisely, then the parachute would fly along the RT toward the DZ with no control inputs required. However, wind estimation is not a precise science. Furthermore, calculation of the CARP relies on less than perfect estimates of the parachute aerodynamics, and the flight crews cannot precisely hit the CARP for each airdrop mission [especially in the case of massive (multiple) deliveries]. Therefore, the AGAS GNC system is used to overcome these potential errors.

The ultimate goal of the AGAS system is to allow delivery aircraft to drop payloads accurately at or above $5500 \mathrm{~m}$, keeping the aircraft out of the range of shoulder-fired ground-to-air missiles. Another benefit of the system is the ability to preaddress each bundle in a load and to guide the individual bundles to their own preprogrammed DZs. Obviously, to accomplish these goals, the AGAS system needs to be simple, affordable, durable, and reusable. (It should survive multiple drops without any repairs.) It should not require major modifications to the standard delivery system's harness or bundle, major modifications to the cargo parachute, or a significant amount of rigger training. The system is required to provide an accuracy of at least $100 \mathrm{~m}$ with a desired goal of 50-m circular error probable. No changes to the parachute or cargo system were allowed.

As a result, the AGAS design concept employs a commercial global positioning system (GPS) receiver and a heading reference as navigation sensors, an inexpensive guidance computer to determine and activate the desired control inputs, and application of four pneumatic muscle actuators (PMAs) to generate control inputs. The navigation system and guidance computer are secured to an existing container delivery system, whereas the PMAs are attached to each of four parachute risers and to the container. Control is affected by lengthening one or two adjacent risers. On deployment of the system from the aircraft, the guidance computer steers the system along the preplanned RT. The AGAS concept relies on sufficient control authority to be produced to overcome errors in wind estimation and in the point of release of the system from the aircraft.

The literature reviewed on the subject showed that precision airdrop activities to date have focused more on the development of 
the decelerator system and less on the development of avionics and technologies required for precision GNC. Furthermore, although autonomous control of the high-glide parafoils has been studied extensively, ${ }^{6-9}$ the literature search produced no references to the autonomous control of a low-glide circular parachute.

The initial work on the development of the AGAS GNC system used a simple three-degree-of-freedom (DOF) model incorporating sensor and actuator dynamics and a simple empirical bang-bang control strategy. 3,4,10,11 Two major objectives were pursued. First was to verify the effectiveness of the predefined-trajectory seeking control strategy with a good wind estimate vs a control strategy that simply seeks the target area (TA) without using any knowledge of the winds. Second was to estimate the impact of changing the characteristics of the sensor suite and actuator dynamics on the overall system performance. The resulting GNC algorithm was successfully tested in simulation. However, preliminary flight tests of this GNC algorithm showed the inadequacy of a 3-DOF model. In particular, certain physical phenomena have been observed that the 3-DOF parachute model could not predict.

The present paper discusses recent advancements in the development of the AGAS system. Specifically, it addresses the issue of theoretical synthesis of the optimal control for the given hardware architecture and analysis of optimal control stability. It also introduces a practical control algorithm motivated by the optimal control law. This practical algorithm was extensively tested in simulations that incorporate a new 6-DOF model of a controlled circular parachute developed by the authors. ${ }^{12}$ The resulting practical control algorithm was finally implemented on an AGAS guidance computer and successfully flight tested. ${ }^{13}$

This paper is organized as follows. Section II contains a brief description of AGAS architecture and of its components. Section III includes the classical synthesis of an optimal control law using Pontrjagin's maximum principle as well as stability analysis of a feedback bang-bang control law motivated by the optimal control analysis. Sections IV and V contain a discussion of the development of the flight control algorithm implemented in simulation and flight. Finally, Sec. VI has a discussion of the setup and results of a final flight test performed at the U.S. Army Yuma Proving Ground, Yuma, Arizona (YPG) in September 2001.

\section{AGAS Architecture and Components}

In general, AGAS may be implemented on any circular parachute. (A flat circular parachute is the one that when laid out on the ground forms a circle.) In this study, a standard G-12 parachute was ultimately employed. This 20 -m-diam parachute, with 64 suspension lines, weighs $60 \mathrm{~kg}$ and is capable of carrying a payload of up to 1 ton in weight with a descent rate of around $9 \mathrm{~m} / \mathrm{s}$ (Ref. 14).

A cargo box that is currently employed for the AGAS is a prototype adopted from the A-22 delivery container. It is suspended from the system (Fig. 1) and houses the GNC system, PMA instrumentation, and parachute control unit (PCU).

The PMAs, developed by Vertigo, Inc., are braided fiber tubes with neoprene inner sleeves that can be pressurized by nitrogen. ${ }^{15}$ On pressurization, the PMA contracts in length from 7.6 to $5.8 \mathrm{~m}$ and expands in diameter. On venting, it does the opposite (lengthens on $30 \%$ ).

When three of four PMAs are pressurized (filled) and one is activated (vented), this action deforms the parachute, creating an asymmetrical shape, essentially shifting the center of pressure, and providing a drive or slip condition. This forces the parachute to glide in the direction opposite the control action (vented PMA). Two adjacent PMAs can be activated simultaneously. Figure 2 shows both possibilities (one and two PMAs activated) realized in a computational fluid dynamics-based simulation ${ }^{\mathbb{a}}$ and observed in the flight test during the airdrops at YPG.

\footnotetext{
"Data available from Mosseev, Y., "Fluid-Structure Interaction Simulation of the U.S. Army G-12 Parachute," Rept. 17-01-RDD Ozon, Contract 68171-01-M-6349, online at http://www.mtu-net.ru/mosseev/rd.html [cited January 2001].
}

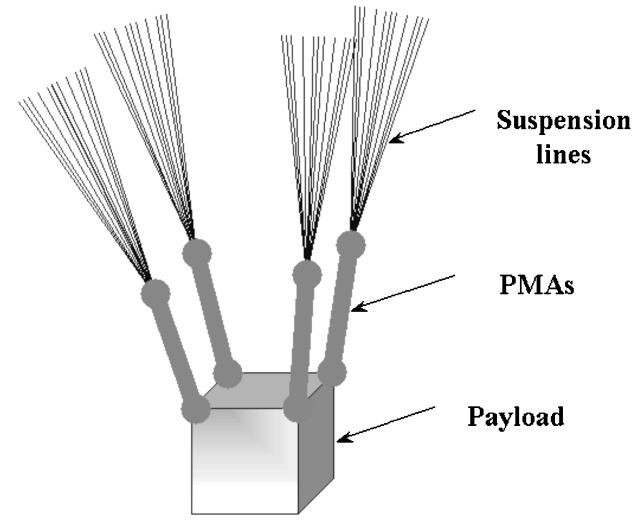

Fig. 1 Scheme of payload suspension.

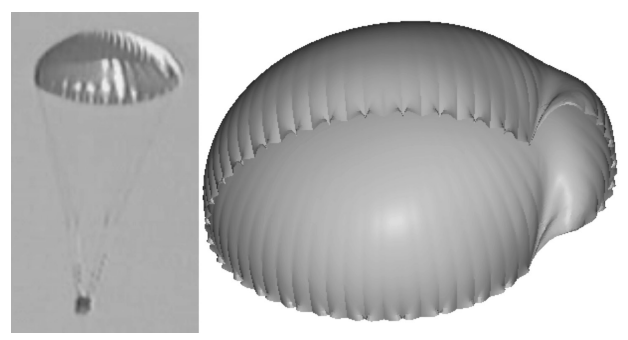

a) One

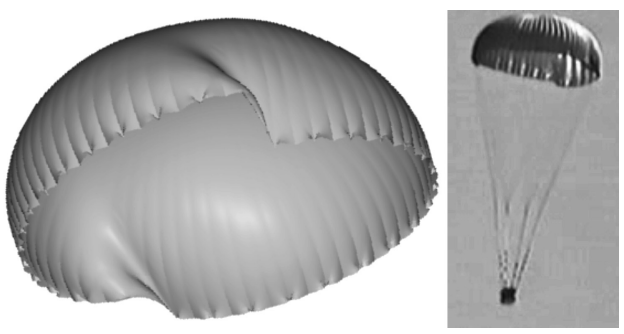

b) Two

Fig. 2 PMAs actuated (vented).

The PCU consists of two accumulator tank valves and pressure circuits and resides in a specially designed container that occupies space atop a cargo container. The volume of the onboard nitrogen tanks limits the number of possible fills for all four PMAs to 32 per drop. PMA fill and vent times remain a constant $5 \mathrm{~s}$ throughout each drop (regardless the of the volume of gas remaining in the tanks). The GNC electronics package, which is installed into a box in the payload, is connected to the PCU.

The full weight of the AGAS package to be added to the standard parachute (including PCU with PMAs, batteries, sensor suite, and GNC computer) is about $80 \mathrm{~kg}$. When fully charged with gas, the system weighs about $11 \mathrm{~kg}$ more.

Circular parachutes have a very limited ability to overcome winds. This underscores the importance of having a reference trajectory that closely matches the flight path of a parachute during an uncontrolled drop. Therefore, there is a strong need to use the latest available wind profile in the DZ to precompute the CARP and RT. Delay in parachute deployment from the time a CARP was computed may cause significant degradation in accuracy, even for the case of a controlled drop. Simulations of uncontrolled drops for the RT computed at hour 0 and of the parachute being released up to $10 \mathrm{~h}$ later ${ }^{16}$ are shown in Fig. 3. Obviously impact points for later releases do not coincide with the impact point for the release at hour 0 (the target point). In fact, for this particular set of wind profiles, touchdown accuracy gradually degraded to more than $3000 \mathrm{~m}$.

\section{Synthesis of the Control Algorithm}

Based on the AGAS concept introduced earlier, the optimal control problem for determination of the parachute trajectories from 


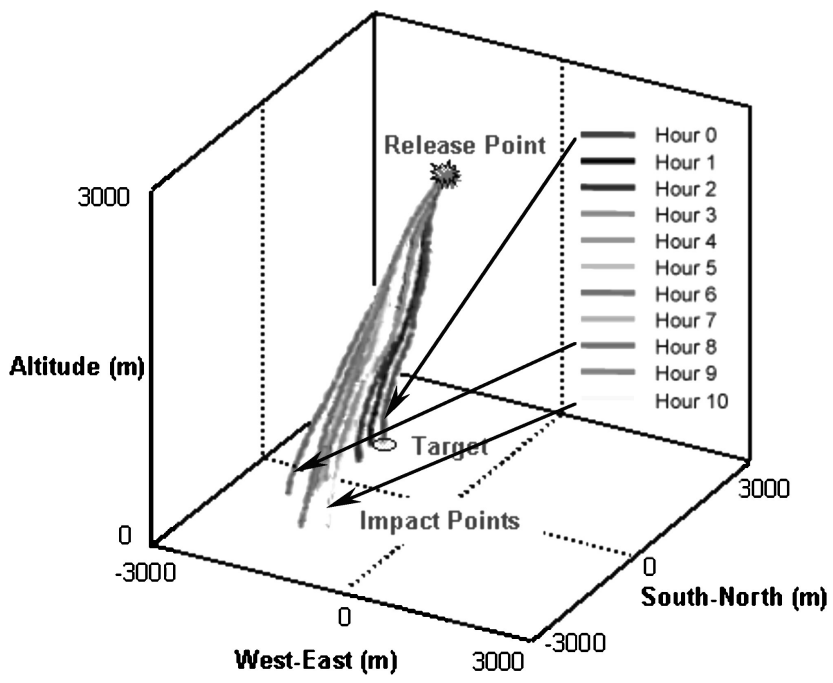

Fig. 3 Uncontrolled trajectories with the different wind profiles.

an actual release point to the DZ can be formulated as follows: Among all admissible trajectories that satisfy the system of differential equations, given initial and final conditions and constraints on control inputs, determine the optimal trajectory that minimizes a cost function of state variables $\boldsymbol{z}$ and control inputs $\boldsymbol{u}$

$$
J=\int_{0}^{T} f_{0}(t, z, \boldsymbol{u}) \mathrm{d} t
$$

and compute the corresponding optimal control.

In Eq. (1), $T$ is a descent time, which is not known a priori, not only because of atmospheric turbulence but also because a descent rate also depends on control state. (Activation of each PMA decreases it by approximately $3 \%$ ). For the AGAS, the most suitable cost function $J$ is the number of PMA activations. However, this cost function cannot be formulated analytically in the form given by expression (1). Therefore, other well-known integrable cost functions were investigated, and the results obtained were used to determine the most suitable cost function for the problem at hand.

To determine the optimal control strategy, Pontrjagin's principle (see Ref. 17) was applied to a simplified kinematic planar (3-DOF) model of the parachute. In this condition, the control objective is to steer the parachute to a single stationary point on a horizontal plane. Obviously this should be done for the final time $t_{f}$ less than $T$.

\section{A. Optimal Control}

The simplest model describing parachute kinematics in the horizontal plane with four equal on-off controllers (Fig. 4) may be written as follows:

$$
\dot{\boldsymbol{P}}=\boldsymbol{R} \boldsymbol{U}, \quad \dot{\psi}=C+\zeta(t)
$$

(If not explicitly defined, bold letters/symbols denote vectors and matrices. If coordinate symbol is omitted, local tangent coordinate frame $\{I\}$ is assumed.) Here $\boldsymbol{P}=[x, y]^{T}$ represents a vector of position errors in the horizontal plane that has to be driven to zero using the control vector $\boldsymbol{U}=[u, v]^{T}$. Furthermore, $\boldsymbol{R}={ }_{B}^{I} \boldsymbol{R}$ is a rotation matrix from the body $\{B\}$ to the local tangent plane (LTP) frame $\{I\}$ defined as

$$
\boldsymbol{R}=\left(\begin{array}{cc}
\cos \psi & -\sin \psi \\
\sin \psi & \cos \psi
\end{array}\right)
$$

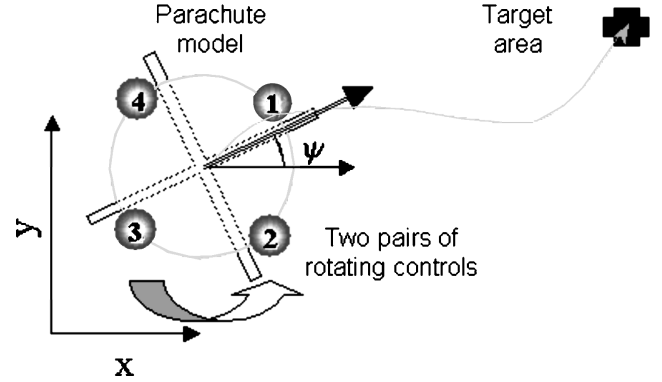

Fig. 4 Projection of the optimization task onto the horizontal plane.

Finally, $\psi$ is a parachute yaw angle (angle between the body $x$ axis and the $x$ axis of the LTP), $C$ is a constant, and function $\zeta(t)$ represents yaw rate disturbance.

This model approximates the impact on the parachute velocity in the lateral plane caused by the activation of each of the four PMAs: $u, v \in[-V ; 0 ; V]$. Specifically for the G-12-based AGAS, $V$ is on the order of $4 \mathrm{~m} / \mathrm{s}$.

The Hamiltonian for system (2) can be written as

$$
H=\left(p_{x}, p_{y}\right) \boldsymbol{R} \boldsymbol{U}+p_{\psi}[C+\zeta(t)]-f_{0}
$$

where differential equations for the adjoint variables $p_{x}, p_{y}$, and $p_{\psi}$ are

$$
\dot{p}_{x}=\dot{p}_{y}=0, \quad \dot{p}_{\psi}=\left(p_{x}, p_{y}\right)\left(\begin{array}{c}
u \sin \psi+v \cos \psi \\
-u \cos \psi+v \sin \psi
\end{array}\right)
$$

We consider two cost functionals,

$$
f_{0} \equiv 1, \quad f_{0} \equiv|u|+|v|
$$

typical for the minimum-time and minimum-fuel problems. For the model given by Eq. (2), the minimum-time problem implies that the parachute must be driven to the origin in minimum time given the constraints on the control vector $\boldsymbol{U}$. Note also that in this application, the second cost function defines the system's momentum or energy rather than fuel because AGAS uses gas only to activate PMAs. (There is no gas expenditure needed to maintain PMA filled/vented).

Importantly, with four on-off controllers available, a circular parachute can move in only one of eight possible directions with respect to the body frame. This makes performance of a control algorithm very sensitive to the rotation of the parachute or lack thereof. Specifically, if the parachute is not rotating, there only exists a single initial condition for which the two-point boundary-value problem can be solved. This is the reason for introducing a non-zero yaw rate in Eq. (2). Furthermore, because the yaw rate can never be precisely known, the disturbance term $\zeta(t)$ was also added. The two main sources of uncertainty in yaw rate include wind disturbance and yaw moment due to adjacent PMA activations (see Sec. IV).

According to Pontrjagin et al., ${ }^{17}$ the optimal control is determined as $\boldsymbol{u}_{\mathrm{opt}}=\operatorname{argmax} H(\boldsymbol{p}, \boldsymbol{z}, \boldsymbol{u})$. Therefore, for the time-minimum problem, the optimal control is given by

$$
\begin{aligned}
& u=V \operatorname{sign}\left[\left(p_{x}, p_{y}\right)\left(\begin{array}{c}
\cos \psi \\
\sin \psi
\end{array}\right)\right] \\
& v=V \operatorname{sign}\left[\left(p_{x}, p_{y}\right)\left(\begin{array}{c}
-\sin \psi \\
\cos \psi
\end{array}\right)\right]
\end{aligned}
$$

Figure 5 shows the graphical interpretation of these expressions. In general, the vector $\left(p_{x}, p_{y}\right)$ defines a direction toward the DZ and establishes a semiplane perpendicular to itself that defines the nature of control actions. Specifically, if the PMA happens to be located within a certain operating angle (OA) $\Delta$ with respect to the vector $\left(p_{x}, p_{y}\right)$, it should be activated. For a time-optimum problem, $\Delta=\pi$. Therefore, two PMAs will always be active as determined by the parachute rotation attitude. (We do not address the case of 


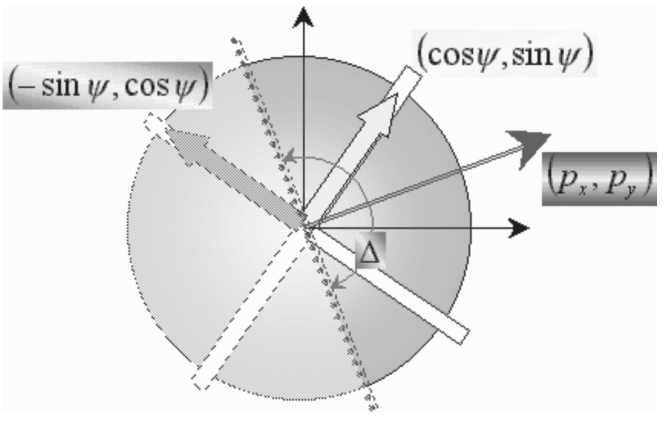

Fig. 5 Time-optimal control.
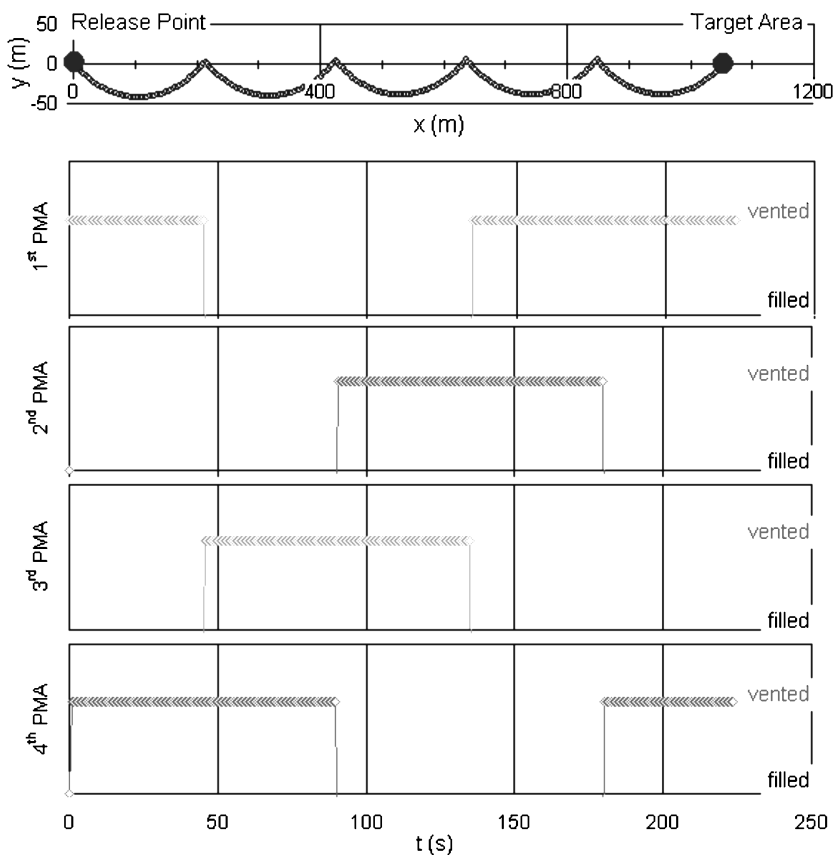

Fig. 6 Example of the time-optimal trajectory and time-optimal controls.
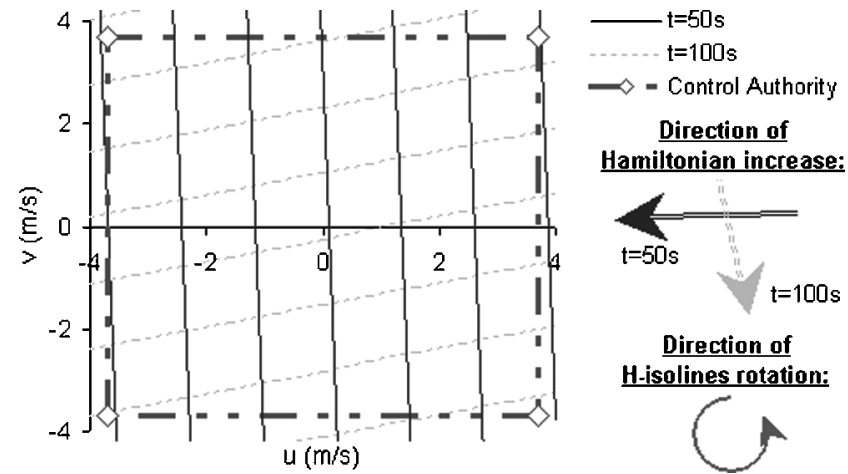

Fig. 7 Hamiltonian isolines for the time-optimal control problem.

singular control, which in general is possible if the parachute is required to satisfy a final condition for yaw angle.)

Figure 6 shows an example of a time-optimal trajectory. It consists of several arcs and a sequence of activations. For the sake of simplicity, $\dot{\psi}=2 \mathrm{deg} / \mathrm{s}$ was taken for this simulation, as observed in one of the earliest flight tests. [In principle because of symmetry, no rotation should be observed unless any kind of asymmetry is introduced.] Figure 7 demonstrates two Hamiltonian isoline patterns corresponding to two instances of time. Because isolines for the time-optimal problem are straight lines rotating counterclockwise with $2-\mathrm{deg} / \mathrm{s}$ angular velocity, the optimal solution on this graph
(Hamiltonian maximum) can be located in one of four corners of the controls envelope.

For the fuel-minimum problem, we obtain analogous expressions for the optimal control inputs:

$$
\begin{gathered}
p_{x} \cos \psi+p_{y} \sin \psi \geq 1 \Rightarrow u=V \\
-p_{x} \sin \psi+p_{y} \cos \psi \geq 1 \Rightarrow v=V \\
-1<p_{x} \cos \psi+p_{y} \sin \psi<1 \Rightarrow u=0 \\
-1<-p_{x} \sin \psi+p_{y} \cos \psi<1 \Rightarrow v=0 \\
p_{x} \cos \psi+p_{y} \sin \psi \leq-1 \Rightarrow u=-V \\
-p_{x} \sin \psi+p_{y} \cos \psi \leq-1 \Rightarrow v=-V
\end{gathered}
$$

In this case, each PMA will be employed only when aligned with the certain direction, meaning $\Delta \rightarrow 0$. Figure 8 shows that in this case Hamiltonian isolines are represented by a rhomboid figure, which maintains an orientation so that one of its vertexes touches the control envelope at $u=0$ (as shown in Fig. 8 for the particular instance of time) or $v=0$.

In general, any cost function other than minimum time will require an OA $\Delta \leq \pi$ (Fig. 9). (Note that any control with $\Delta<0.5 \pi$ may not work at all if the parachute is not rotating.)

Figure 10 shows the effect of the OA's magnitude on the flight time, fuel, and number of PMA activations (from vented to filled state). Note that the nature of the dependence of the number of activations on the OA is the same as that of the time of flight. This implies that by solving the time-minimum problem, we automatically ensure a minimum number of activations. Moreover, it is also seen that the slope of these two curves in the interval $\Delta \in[0.5 \pi ; \pi]$ is flat. This implies that small changes of OA from its optimal value will result in negligible impact on the number of activations. Therefore, changing the OA to account for the realistic PMA model, as is done on AGAS (Sec. IV), will not change the number of activations significantly.

Figure 11 demonstrates the influence of constant yaw rate on different OAs. The results were obtained for the time-optimal control problem shown in Fig. 6. Obviously, the smaller the yaw rate is, the

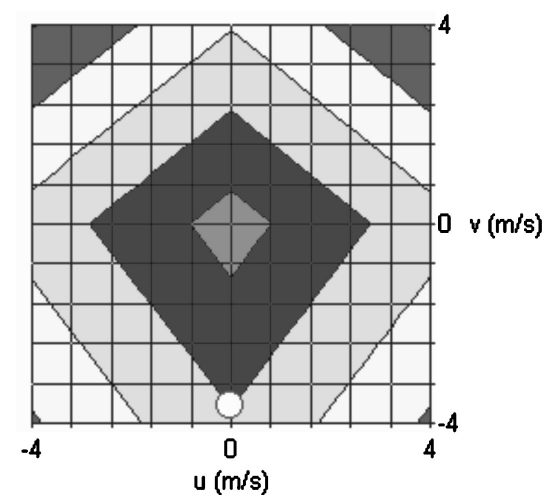

Fig. 8 Hamiltonian isolines for the fuel-minimum problem.

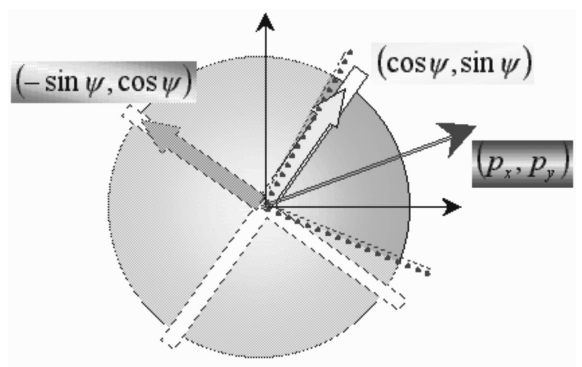

Fig. 9 Generalized case of optimal control. 


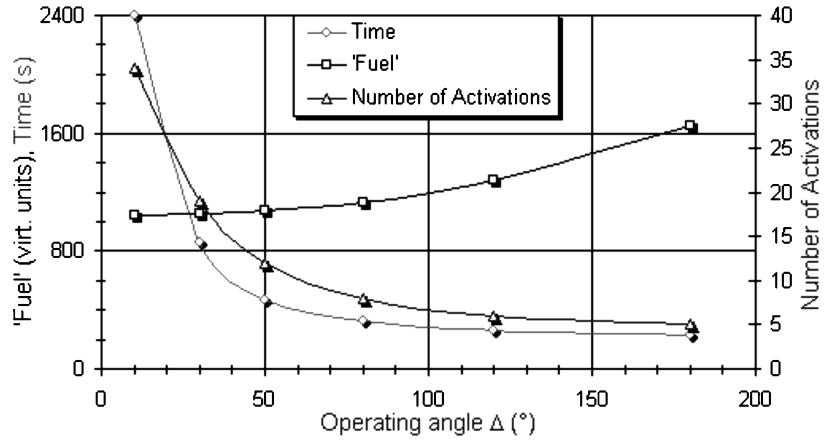

Fig. 10 Influence of the OA's magnitude.

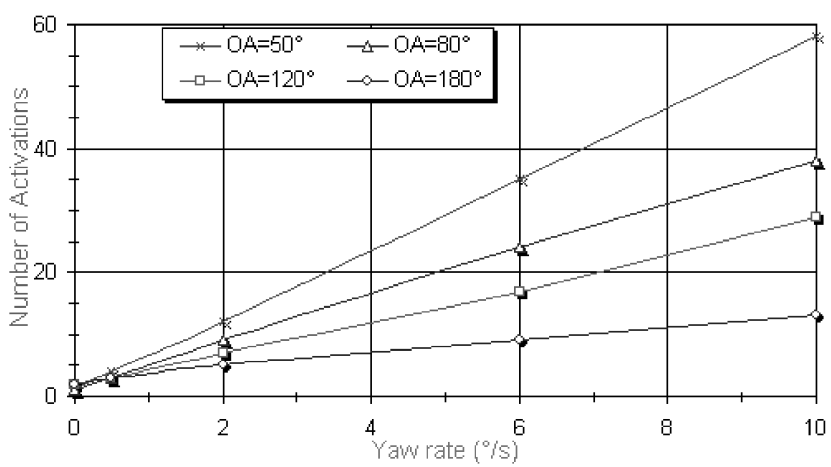

Fig. 11 Influence of a constant yaw rate.
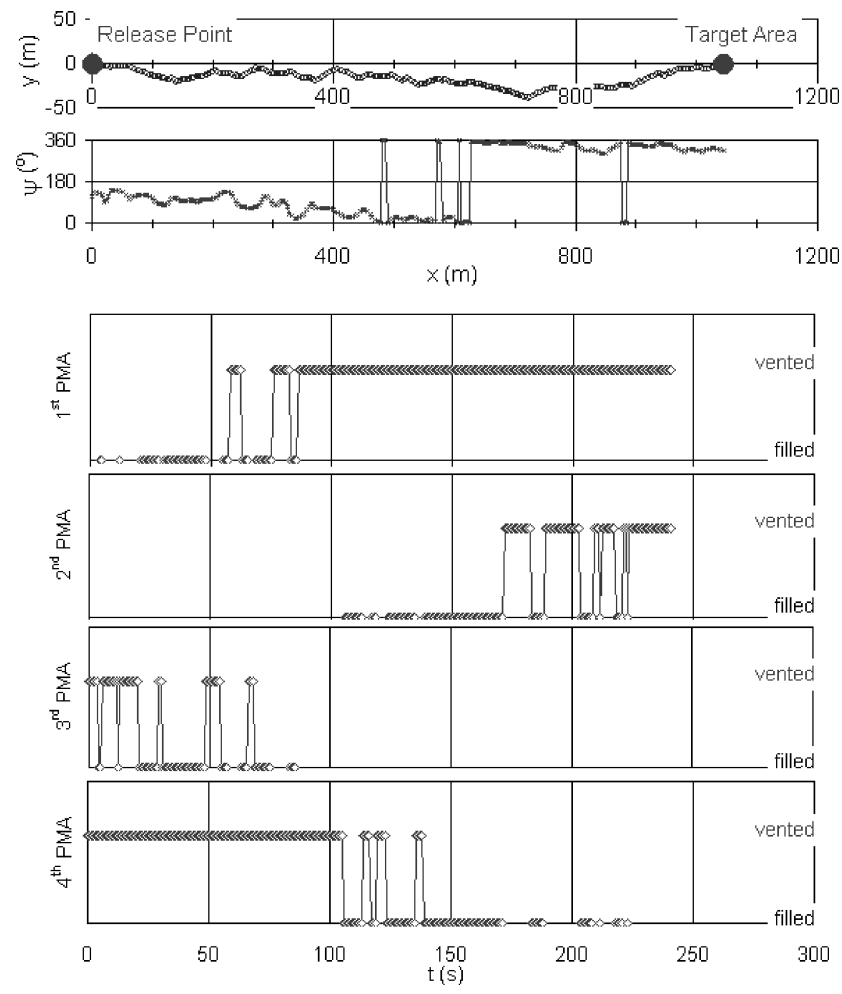

Fig. 12 Flight path computed with usage of a real yaw angle profile.

smaller the number of activations. Decreasing the OA for the same yaw rate leads to an increase in the number of PMA activations.

Figure 12 includes simulation results for the case where yaw angle from a flight test was used to drive the first equation in Eq. (2), whereas optimal control was computed using Eq. (7). As can be seen, the flight-test yaw angle is not smooth. Neither is it monotonic. Although a synthesized optimal control drives the model of the parachute toward TA, because of the erratic yaw the number of
PMA activations increases to 35 (vs 12 with the monotonic 2-deg/s yaw rate as seen from Fig. 10). For this particular simulation, the OA was equal to $2.5 \mathrm{rad}$. This example illustrates the sensitivity of the optimal control algorithm to uncertainties in yaw angle. Therefore, the flight control algorithm must be more robust to these uncertainties to prevent a significant increase in the number of PMA activations.

\section{B. Stability Analysis}

The minimum-time optimal control strategy obtained in the preceding section motivates the following feedback control law (7) for the system of equations (2),

$$
\boldsymbol{U}=-\operatorname{sign}\left(\boldsymbol{R}^{T} \boldsymbol{P}\right)
$$

where, for any vector $\rho \in \mathfrak{R}^{n}$, we define $\operatorname{sign} \rho=$ $\left[\operatorname{sign} \rho_{1}, \ldots, \operatorname{sign} \rho_{n}\right]^{T}$.

It is easy to show using Lyapunov stability theory that this control strategy is globally asymptotically stable. Let

$$
L=\boldsymbol{P}^{T} \boldsymbol{P}
$$

denote the Lyapunov function for the feedback system

$$
G=\left\{\begin{array}{c}
\dot{\boldsymbol{P}}=\boldsymbol{R} \boldsymbol{U}, \\
\boldsymbol{U}=-\operatorname{sign}\left(\boldsymbol{R}^{T} \boldsymbol{P}\right)
\end{array}\right.
$$

Then

$$
\dot{L}=\boldsymbol{P}^{T} \dot{\boldsymbol{P}}+\dot{\boldsymbol{P}}^{T} \boldsymbol{P}=-2 \boldsymbol{P}^{T} \operatorname{Rsign}\left(\boldsymbol{R}^{T} \boldsymbol{P}\right)
$$

Because $\boldsymbol{\rho}^{T} \operatorname{sign} \boldsymbol{\rho}>0$, for any nonzero vector $\boldsymbol{\rho}$, and because $\boldsymbol{R}$ is a rotation matrix, we conclude that $\dot{L}<0, \forall \boldsymbol{P} \neq \mathbf{0}$.

As discussed in Sec. III, the time-optimal control strategy corresponds to the OA of $180 \mathrm{deg}$. The same is true for the feedback control strategy (9). Additional design considerations presented in Sec. IV have resulted in a control strategy with an OA that is less than $180 \mathrm{deg}$.

Therefore, in the remainder of this section we propose to analyze stability of a control strategy that uses a smaller OA. To do this, we define a new function $\operatorname{sign}_{\triangle}: \mathfrak{R}^{n} \times \mathfrak{R}^{n} \rightarrow \mathfrak{R}^{1}$ as follows. Let $\rho_{1}$ and $\rho_{2}$ be any two vectors in $\Re^{n}$. Then,

$$
\operatorname{sign}_{\Delta} \arg \left(\boldsymbol{\rho}_{1}, \boldsymbol{\rho}_{2}\right)=\left\{\begin{array}{cc}
1, & 2 \arg \left(\boldsymbol{\rho}_{1}, \boldsymbol{\rho}_{2}\right) \leq \Delta \\
0, & \left|2 \arg \left(\boldsymbol{\rho}_{1}, \boldsymbol{\rho}_{2}\right)-\pi\right|<\Delta \\
-1, & \text { otherwise }
\end{array}\right.
$$

Now using Eq. (13), we define the feedback control strategy (Fig. 13)

$$
\boldsymbol{U}=\left\{\begin{array}{lc}
{\left[\operatorname{sign}_{\Delta} \arg \left(\frac{\boldsymbol{R}^{T} \boldsymbol{P}}{\left\|\boldsymbol{R}^{T} \boldsymbol{P}\right\|},\left[\begin{array}{l}
1 \\
0
\end{array}\right]\right)\right.} \\
-\operatorname{sign}_{\Delta} \arg \left(\frac{\boldsymbol{R}^{T} \boldsymbol{P}}{\left\|\boldsymbol{R}^{T} \boldsymbol{P}\right\|},\left[\begin{array}{l}
0 \\
1
\end{array}\right]\right) & \\
\mathbf{0}, & \text { otherwise }
\end{array}\right.
$$

Clearly, for $\Delta=\pi$, Eq. (14) reduces to Eq. (9). Let the Lyapunov function $L$ be defined in Eq. (10). Then

$$
\dot{L}=-2 \boldsymbol{P}^{T} R\left[\begin{array}{c}
-\operatorname{sign}_{\Delta} \arg \left(\frac{\boldsymbol{R}^{T} \boldsymbol{P}}{\left\|\boldsymbol{R}^{T} \boldsymbol{P}\right\|},\left[\begin{array}{l}
1 \\
0
\end{array}\right]\right) \\
-\operatorname{sign}_{\Delta} \arg \left(\frac{\boldsymbol{R}^{T} \boldsymbol{P}}{\left\|\boldsymbol{R}^{T} \boldsymbol{P}\right\|},\left[\begin{array}{l}
0 \\
1
\end{array}\right]\right)
\end{array}\right]
$$

Define a function $\operatorname{sign}_{\varepsilon}: \Re^{1} \rightarrow \Re^{1}$ analogous to Eqs. (13)

$$
\operatorname{sign}_{\varepsilon} a=\left\{\begin{array}{lc}
1, & a \geq \varepsilon \\
0, & |a|<\varepsilon \\
-1, & a \leq-\varepsilon
\end{array}\right.
$$


Fig. 13 Illustration of the control strategy in terms of function (13).

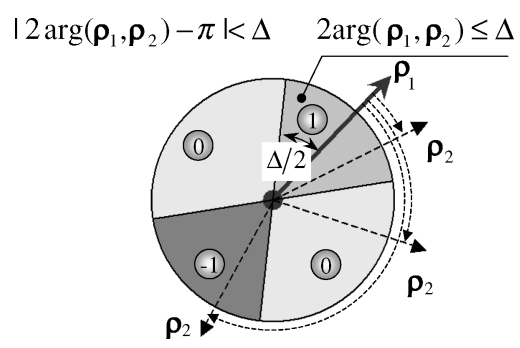

Fig. 14 Possibility of absence of asymptotic stability.

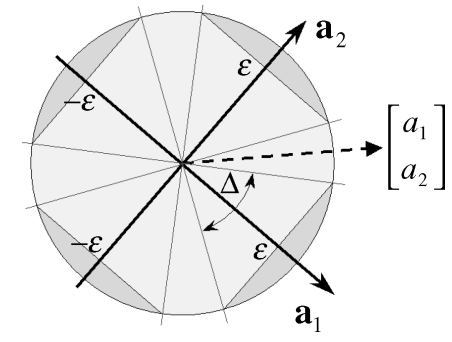

Let

$$
\begin{gathered}
a_{1}=\left\{\left(\frac{\boldsymbol{R}^{T} \boldsymbol{P}}{\left\|\boldsymbol{R}^{T} \boldsymbol{P}\right\|}\right)^{T},\left[\begin{array}{l}
1 \\
0
\end{array}\right]\right\} \\
a_{2}=\left\{\left(\frac{\boldsymbol{R}^{T} \boldsymbol{P}}{\left\|\boldsymbol{R}^{T} \boldsymbol{P}\right\|}\right)^{T},\left[\begin{array}{l}
0 \\
1
\end{array}\right]\right\}, \quad \varepsilon=\cos \left(\frac{\Delta}{2}\right)
\end{gathered}
$$

Then (Fig. 14)

$$
\begin{gathered}
\operatorname{sign}_{\Delta} \arg \left(\frac{\boldsymbol{R}^{T} \boldsymbol{P}}{\left\|\boldsymbol{R}^{T} \boldsymbol{P}\right\|},\left[\begin{array}{l}
1 \\
0
\end{array}\right]\right\}=\operatorname{sign}_{\varepsilon} a_{1} \\
\operatorname{sign}_{\Delta} \arg \left(\frac{\boldsymbol{R}^{T} \boldsymbol{P}}{\left\|\boldsymbol{R}^{T} \boldsymbol{P}\right\|},\left[\begin{array}{l}
0 \\
1
\end{array}\right]\right)=\operatorname{sign}_{\varepsilon} a_{2}, \quad \boldsymbol{R}^{T} \boldsymbol{P}=\left\|\boldsymbol{R}^{T} \boldsymbol{P}\right\|\left[\begin{array}{l}
a_{1} \\
a_{2}
\end{array}\right]
\end{gathered}
$$

Furthermore, for $\forall \boldsymbol{P} \neq \mathbf{0}$,

$$
\dot{L}=-2\left\|\boldsymbol{R}^{T} \boldsymbol{P}\right\|\left[\begin{array}{ll}
a_{1} & a_{2}
\end{array}\right]\left[\begin{array}{l}
d \operatorname{sign}_{\varepsilon} a_{1} \\
d \operatorname{sign}_{\varepsilon} a_{2}
\end{array}\right]=M\left\|\boldsymbol{R}^{T} \boldsymbol{P}\right\|
$$

where

$M=$

$\begin{cases}-4, & a_{1} \geq \varepsilon, \quad a_{2} \geq \varepsilon \vee a_{1} \leq-\varepsilon, \quad a_{2} \leq-\varepsilon \\ -2, & -\varepsilon<a_{1}<\varepsilon, \quad a_{2} \geq \varepsilon \vee a_{1} \geq \varepsilon, \quad-\varepsilon<a_{2}<\varepsilon \\ -2, & -\varepsilon<a_{1}<\varepsilon, \quad a_{2} \leq-\varepsilon \vee a_{1} \leq-\varepsilon, \quad-\varepsilon<a_{2}<\varepsilon \\ 0, & \left|a_{1}\right|<\varepsilon, \quad\left|a_{2}\right|<\varepsilon\end{cases}$

The last expression indicates that the candidate Lyapunov function $L=\boldsymbol{P}^{T} \boldsymbol{P}$ does not guarantee asymptotic stability for the feedback control strategy (14) when $\left|a_{1}\right|<\varepsilon$ and $\left|a_{2}\right|<\varepsilon$. As illustrated in Fig. 14, this situation can occur only when $\Delta<0.5 \pi$.

Therefore, the feedback strategy (14) guarantees global asymptotic stability in the sense of Lyapunov for any $\Delta \geq 0.5 \pi$.

\section{Flight Control Algorithm}

As discussed in Sec. II, the activation box for PMAs is capable of only bang-bang control. Optimal control analysis of a simplified parachute model discussed in Sec. III suggested that for the cost functions considered, bang-bang is also the optimal control strategy. Furthermore, this analysis led to an important concept of an OA, which was used to define the basic control concept for AGAS. Because for a given OA the bang-bang control strategy was shown to minimize the number of activations for a planar model, this strategy was employed to get the parachute to within a predefined altitudedependent TA (defined by inner and outer cones discussed next) and then for the remainder of descent to stay within this area. In addition, this basic strategy must be made robust to uncertainties in yaw motion. (As mentioned earlier, a sensor suite was installed on top of a payload, so that some discrepancy exists between the measured heading reference and the real attitude of the canopy.) These considerations were used to develop the flight control algorithm for AGAS and are detailed next.

\section{A. Basic Control Architecture}

When the relatively low-glide ratio (GR) of circular parachutes is considered, the AGAS can only overcome less than $4-\mathrm{m} / \mathrm{s}$ wind. Therefore, it is imperative that the control system steers the parachute along a prespecified RT obtained from most recent wind prediction. This can be done by comparing the current GPS position of the parachute with the desired one on the RT at a given altitude $h$ to obtain the position error

$$
\boldsymbol{P}(h)=\boldsymbol{P}_{\mathrm{AGAS}}(h)-\boldsymbol{P}_{\mathrm{RT}}(h)
$$

This position error $\boldsymbol{P}(h)$ is computed in LTP frame with an origin in the TA and is then converted to the body axis using an Euler angle rotation $\boldsymbol{R}^{T}$. The resulting body-axis error vector

$$
\boldsymbol{P}_{B}=\boldsymbol{R}^{T} \boldsymbol{P}
$$

is then used to identify the error angle (EA) $\lambda$

$$
\lambda=\arg \boldsymbol{P}_{B}
$$

As opposed to parafoils, circular parachute angular dynamics is characterized by coning motion. Coning motion is represented by coupled oscillations in pitch and roll. Therefore, when averaged over one period, pitch and roll angles are zero, and it is sufficient to use heading angle only when computing rotation matrix $\boldsymbol{R}$ in Eq. (18).

In turn, the EA is then used to define what PMA, $i=1, \ldots, 4$ must be activated:

$$
i=\left\{\begin{array}{ccc}
1, & \text { if } & \lambda \leq(\Delta / 2) \vee \lambda \geq 2 \pi-(\Delta / 2) \\
2, & \text { if } & \lambda \in[(3 \pi / 2)-(\Delta / 2) ;(3 \pi / 2)+(\Delta / 2)] \\
3, & \text { if } & \lambda \in[\pi-(\Delta / 2) ; \pi+(\Delta / 2)] \\
4, & \text { if } & \lambda \in[(\pi / 2)-(\Delta / 2) ;(\pi / 2)+(\Delta / 2)]
\end{array}\right.
$$

[By definition $\lambda$ is counted from PMA 3 counterclockwise, that is, in the situation shown as an example in Fig. 15, PMAs 2 and 3 would be activated (vented)].

As suggested in Sec. III, to account for the refill time and sensor errors, the OA was set to $\Delta \approx 2.5 \mathrm{rad}$ instead of $\Delta=\pi$. (On the earliest AGAS versions, refill time was not constant and was equal to about $20 \mathrm{~s}$ at the end; that for the yaw rate of $2 \mathrm{deg} / \mathrm{s}$ gives around 40 deg.) This still allows the activation of a single control input or two simultaneous control inputs without significant degradation of AGAS performance (Fig. 10). Furthermore, it is greater than $\pi / 2$ and, therefore, within the stability range for the OA.

Fig. 15 Control-activation rule.

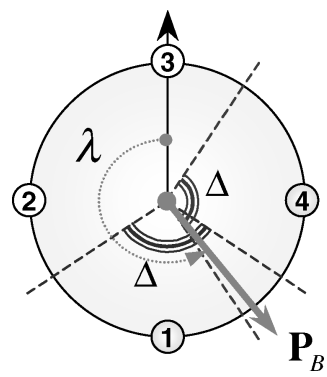




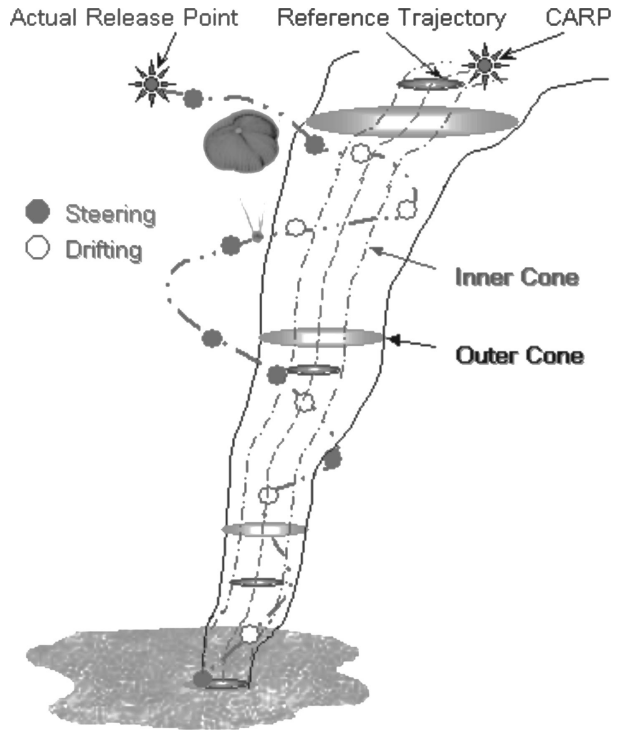

Fig. 16 Outer and inner cones.

\section{B. Outer and Inner Cones}

First, the initial error after deployment should not exceed a certain value because of AGAS's limited control authority. This area of attraction has the radius $R_{A}$ around the RT that can be roughly estimated by a simple formula:

$$
R_{A}(h)=0.8 k_{\Delta} \mathrm{GR}_{\max } h
$$

where $k_{\Delta} \approx \Delta \pi^{-1}$. The coefficient $k_{\Delta}$ is approximated by using the data given in Fig. 10, GR $\mathrm{max}_{\text {ax }}$ for the G-12-based AGAS with two adjacent PMAs activated is equal to 0.52 (Ref. 13), and the coefficient 0.8 accounts for real-world yaw profile.

To eliminate unnecessary activations of PMAs, a tolerance (outer) cone was established (Fig. 16). ${ }^{4}$ Its radius at the CARP (at an altitude of $3000 \mathrm{~m})$ is $\Re_{\text {outer }}(3000)=200 \mathrm{~m}$, and it decreases linearly to $\Re_{\text {outer }}(0)=100 \mathrm{~m}$ radius circle at the TA (at ground level). Should the magnitude of the position error in the lateral plane $\left|\boldsymbol{P}_{B}(h)\right|$ be outside of this tolerance cone,

$$
\left|\boldsymbol{P}_{B}(h)\right|>\Re_{\text {outer }}(h)
$$

a control is activated to steer the system back to the planned RT.

When the system is within the inner cone $\Re_{\text {inner }}$

$$
\left|\boldsymbol{P}_{B}(h)\right|<\Re_{\text {inner }}
$$

(which is set to $60 \mathrm{~m}$ radius regardless of altitude) the control is disabled, and the parachute drifts with the wind $\left(\Re_{\text {inner }}\right.$ was selected to account for the refill time) until the outer cone is reached and control is activated again. These inner and outer cones can be interpreted as altitude-dependent hysteresis surfaces.

The basic control strategy uses the following activation rule: Both the tolerance cone and the operating angle constraints must be active for a given PMA to be activated.

\section{Robustness Issues}

The control algorithm just outlined was flight tested at YPG. As expected, the number of commands to activate PMAs was unacceptably high. This resulted in a premature emptying of onboard nitrogen tanks, thus, leaving the AGAS with no control authority at the bottom of descent. Analysis of flight-test data indicated that this was caused by frequent yaw angle changes and that these changes occurred when one of the adjacent PMAs was actuated while the other one was in transition from vent to full or vice versa.

Figure 17 explains this phenomenon. If one PMA is activated (vented), and an adjacent PMA is performing a transition from one state to another, this causes a yaw moment $\boldsymbol{M}_{c}$. This moment can be useful (when the direction of rotation of the vector $\boldsymbol{P}_{B}$ is opposite to the direction of $\boldsymbol{M}_{c}$ ), or harmful (vice versa). In the latter case, the rotation of the parachute under the action of $\boldsymbol{M}_{c}$ causes a deactivation

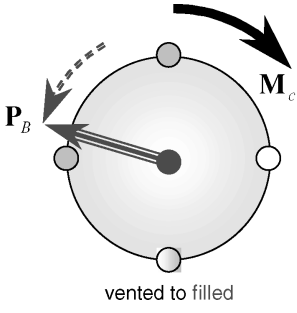

a) Positive

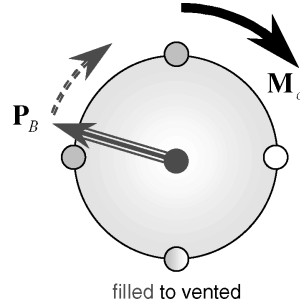

b) Negative
Fig. 17 Effect of PMA transition moment.

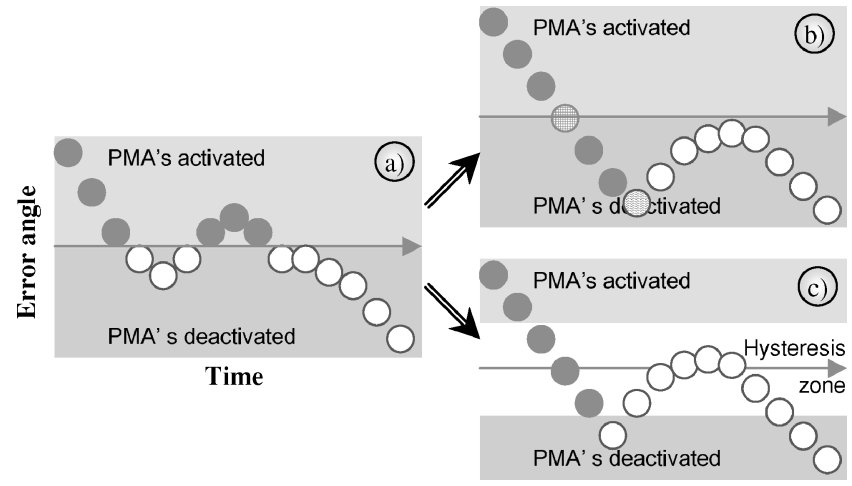

Fig. 18 Two ways of decreasing the influence of yaw oscillations.

command to the PMA that was just activated. Moreover, during this deactivation the useful moment in turn makes the situation even worse. This case is shown in Fig. 18a, where shaded circles denote activated state of any PMA.

To eliminate unnecessary activations, delay logic was introduced in each PMA channel (Fig. 18b). Any new command that requires change in the PMA state triggers the delay timer (circle with a grid inside in Fig. 18b). While the delay timer is active, no command is executed including the triggering command. At the end of the delay, the timer is reset, and the first available command (circle with waves inside in Fig. 18b) is executed until the next command that requires change in the PMA state triggers the delay timer again. (This simple logic was used in lieu of a filter due to programming constraints.)

Another approach that helps reduce the number of unnecessary activations and that meets programming constraints is to use hysteresis as shown in Fig. 18c. The size of a hysteretic zone is adjusted to exclude recurring activations.

Both delay and hysteresis angle values can be adjusted as a function of system dynamics, and, in principle, they achieve the same result.

\section{Prediction Term}

Another approach that drastically improves robustness in the presence of yaw oscillations is to introduce a derivative term into the control logic (18) as follows.

First, change inequalities (22) and (23) to

$$
\left|\boldsymbol{R}^{T}\left(\boldsymbol{P}-k_{c} \dot{\boldsymbol{P}}\right)\right|>\Re_{\text {outer }}, \quad\left|\boldsymbol{R}^{T}\left(\boldsymbol{P}-k_{c} \dot{\boldsymbol{P}}\right)\right|<\Re_{\text {inner }}
$$

where coefficient $k_{c}$ should be adjusted to provide better performance (smallest overshoot). This softens the outer and inner cone edges. For example, if AGAS is approaching the inner cone with high planar velocity rather than slowly drifting into it, it is better to deactivate (fill) all PMAs earlier than would be done by the control strategy based on inequality (23). On the other hand, if AGAS is leaving the outer cone with a high planar velocity, it is worth activating (venting) appropriate PMA(s) earlier than it would be done by inequality (22) to prevent further rapid increase of the radial error.

Second, redefine the EA to be

$$
\lambda=\arg \left\{\boldsymbol{P}^{T}\left(\boldsymbol{P}+k_{r} \dot{\boldsymbol{P}}\right)\right\}
$$

where $k_{r}$ determines the delay in the execution of next command defined by inequality (22), similar to the one discussed in the preceding 

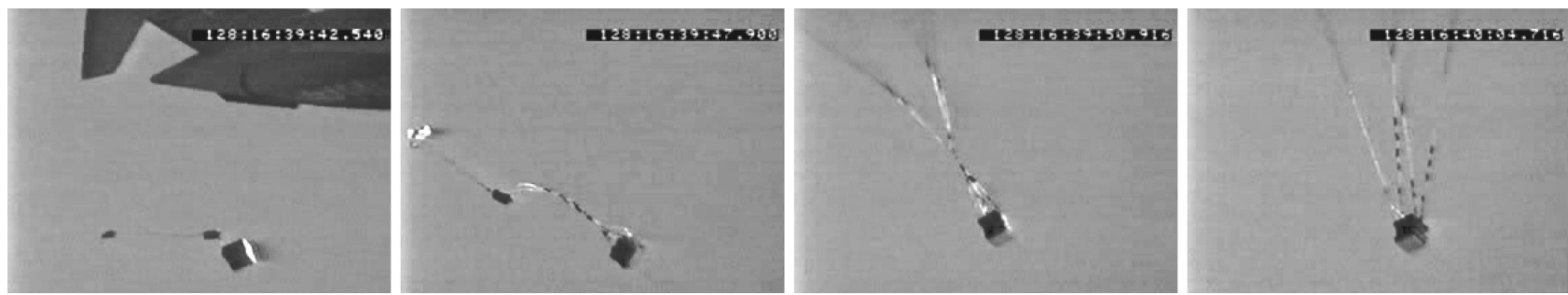

Fig. 19 AGAS deployment and risers untwisting sequence.

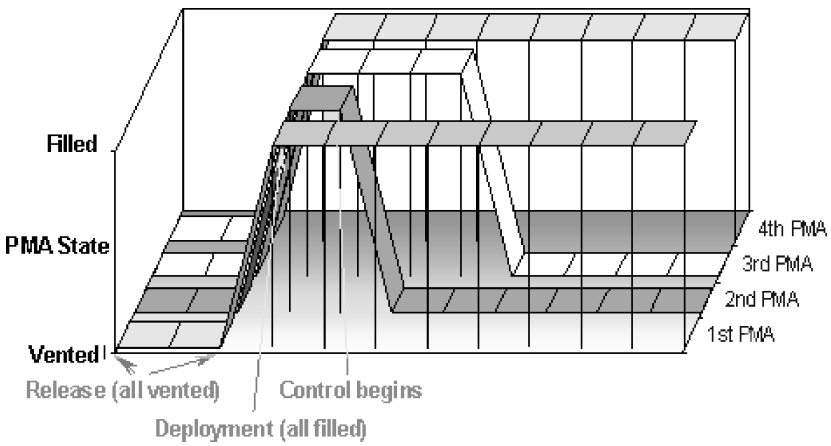

Fig. 20 Control actions history right after release.

section. Compare the definition of the EA in Eq. (25) with that in Eq. (19).

Notice that expressions (24) and (25) have opposite signs for the derivative term. In expression (24), the negative sign accelerates control action, whereas in expression (25), it does the opposite, therefore reducing sensitivity to the oscillations in yaw.

\section{E. Target-Seek Strategy}

For the case when available wind prediction is either too old or nonexistent, an alternative to tracking an RT is proposed. For this purpose, Eq. (17) should be replaced by

$$
\boldsymbol{P}(h)=\boldsymbol{P}_{\mathrm{AGAS}}(h)-k_{w} \boldsymbol{P}_{\mathrm{RT}}(h)
$$

When wind prediction is available, $k_{w}=1$. When wind prediction is either too old or nonexistent, $k_{w}=0$, Eq. (26) becomes

$$
\boldsymbol{P}(h)=\boldsymbol{P}_{\mathrm{AGAS}}(h)
$$

An appropriate value of $k_{w}$ can be determined by comparing realtime motion of AGAS during a drop with its predicted response generated by the onboard model. In fact, assuming the model is sufficiently accurate, it can be used to determine errors in the predicted wind profile.

\section{F. Deployment Delay}

As a safety precaution, the GNC system starts implementing control commands $25 \mathrm{~s}$ after the initial deployment. This time is needed for the AGAS to be released, the main canopy to be fully deployed, and risers to be untwisted, as shown in Fig. 19.

By design, the initial shock during deployment is absorbed by Kevlar $^{\circledR}$ load lines. Thus, all PMAs are initially vented. (When vented they are longer than Kevlar load lines.) The first command sent and executed after the 25-s deployment delay is to fill all PMAs (Fig. 20). After an additional 5 s, any other command can be executed.

\section{Nonlinear Simulation Results}

\section{A. AGAS Model}

The control algorithm discussed in Sec. IV was first tested in a simulation environment using an AGAS model developed in Ref. 12. This model assumes a low-speed descent (with the main canopy fully deployed) and is a complete nonlinear 6-DOF model of a controlled G-12 parachute. This simulation also included a model of the PMA

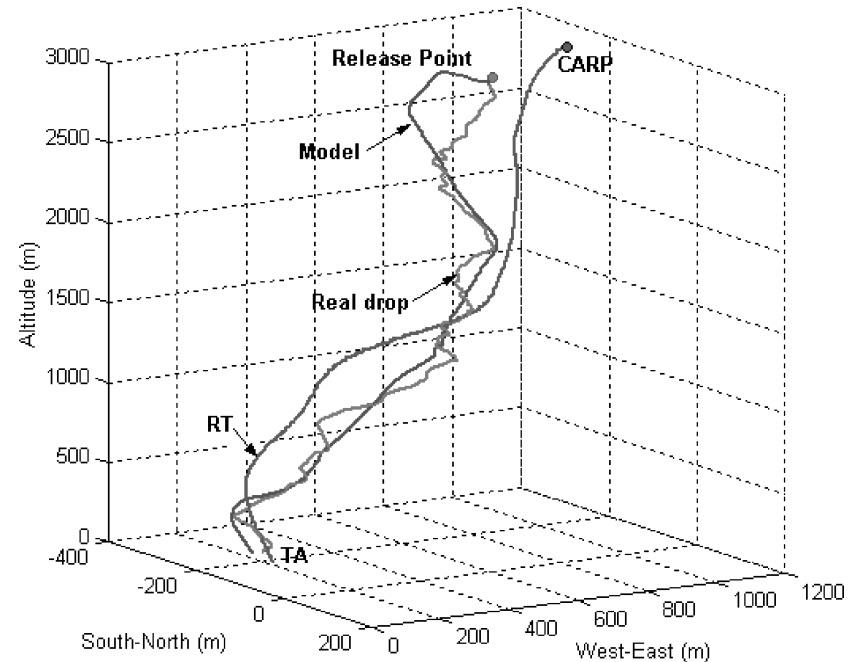

Fig. 21 AGAS model vs real AGAS drop.

dynamics. Figure 21 shows an example where the three-dimensional position of AGAS from a flight test is compared to that generated by the model. The model output matches flight-test data fairly well, with only a $15-\mathrm{m}$ difference between impact points (IPs). The number of PMA activations is the same and is equal to 14 .

\section{B. Simulation Analysis}

Extensive simulation analysis was done to test the flight control algorithm, to determine the accuracy requirements for the sensor suite and the control authority requirements for PCU, and to estimate AGAS overall performance. Some of these results are given next.

Figure 22 showns the influence of the OA's magnitude on control performance. In this case, only the basic control algorithm was tested, that is, no cones, delay, hysteresis, or any other additional features designed to minimize the number of activations discussed in the preceding section were included. Each graph represents radial error vs current altitude during the simulated drops. The target is at $(0, \mathrm{~s} 0)$ on the graph.

Whereas simulation with the $\mathrm{OA}=180 \mathrm{deg}$ ensures the best accuracy of the predefined RT tracking, it also requires 53 activations. (Compare this with 35 activations obtained in simulation with the same yaw profile in Fig. 14 when using 3-DOF model.) With the decrease of $\mathrm{OA}$, the number of activations also decreases ( 17 for $\Delta=90 \mathrm{deg}$ and 14 for $\Delta=60 \mathrm{deg}$ ). However, touchdown accuracy degrades as well.

The grayscale bar in the top-left portion of Fig. 22 indicates whether any PMA was activated during the simulated drop for each OA. Obviously with $\Delta<90 \mathrm{deg}$ (top strip), blind sectors with no PMA activation becomes possible (see Sec. III).

Figure 23 shows the impact of introducing outer and inner cones on the control performance. The operating angle in this simulation and hereafter was $\Delta=143 \mathrm{deg}$, and the number of activations was 9 (as opposed to 24 without cones). Obviously this was achieved by not activating PMAs while between cones. (See the bar similar to the one in Fig. 22 in the top-left corner of Fig. 23). This also results in a slight degradation of the touchdown accuracy. (From about $700 \mathrm{~m}$ 
above DZ, the wind blew the parachute away from TA; however, neither PMA was activated because the parachute was inside the outer cone.)

Although nine activations seem to be an excellent result (for a given CARP generated using the most recent wind profile), further analysis revealed that almost half of the activations were due to yaw oscillations. As discussed in the preceding section, unnecessary activations can be reduced by introducing delay or hysteresis.

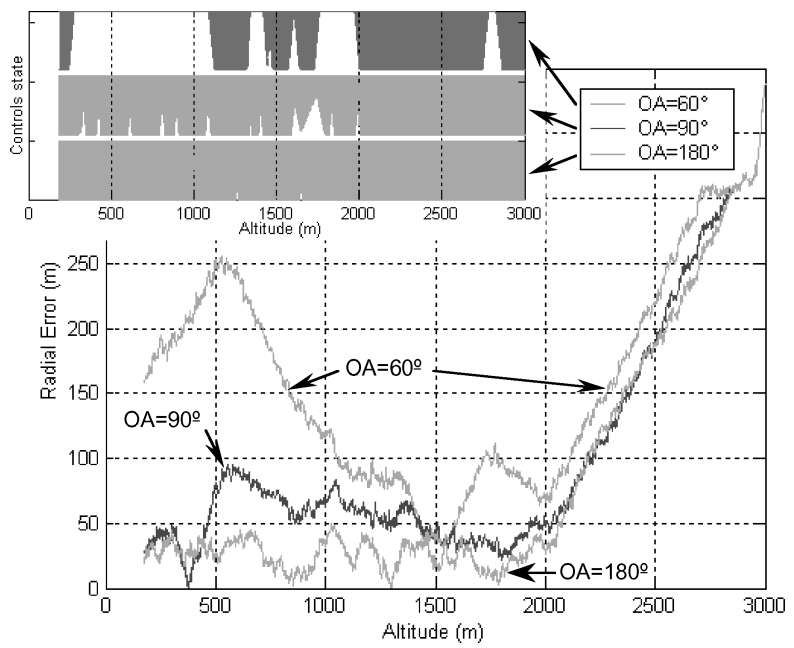

Fig. 22 Simulations with different $\Delta$ (OAs)

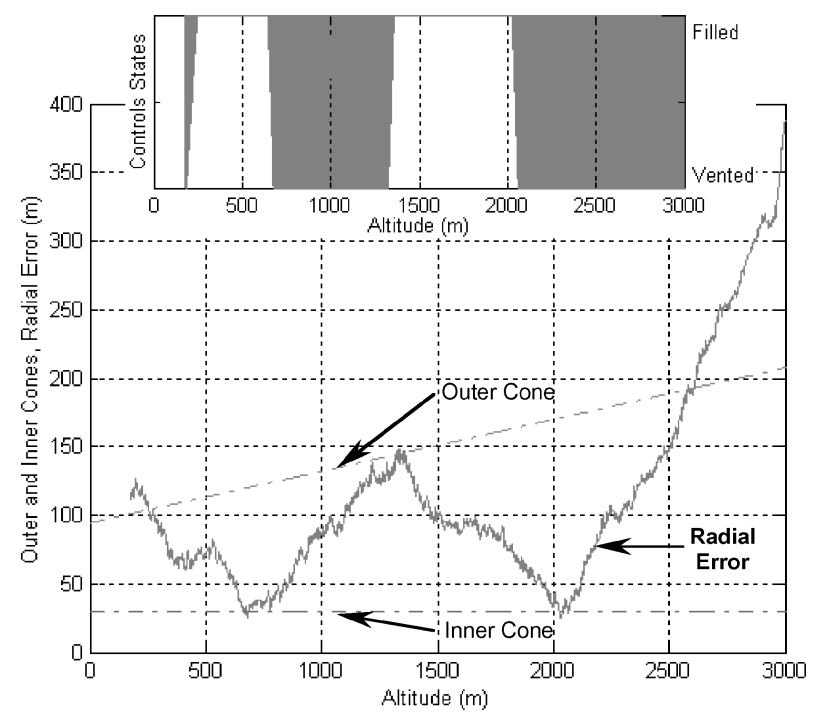

Fig. 23 Introducing the cones.

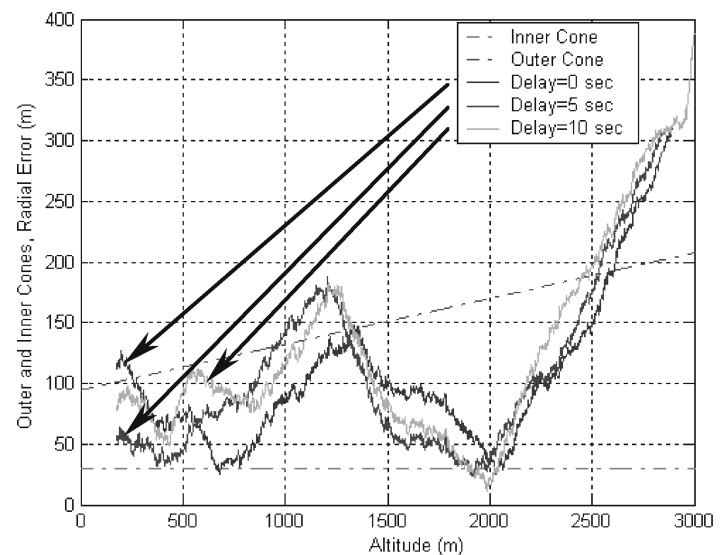

a)
Figure 24 gives an idea of how these features affect performance of the system. In addition, it was discovered that although the delay does not affect the number of PMA activations (for this simulation, it remained approximately the same 9, 9, and 10 for 0-, 5- and 10-s delay, respectively), the EA hysteresis not only improves performance but decreases the number of activations as well: 9 for 0 deg, and 5 for \pm 5 and $\pm 10 \mathrm{deg}$. Therefore, all of the following simulation results include the EA hysteresis of $\pm 5 \mathrm{deg}$.

Figure 25 shows the effect of introducing a prediction term (24). As expected, the overall performance improves. (Trajectory stays strictly between cones.) However, this term leads to a certain increase in the number of PMA activations. (The dead zone between two soft-edge cones is smaller then between solid-edge ones.) For this particular simulation, the number of activations was equal to five with no prediction $k_{c}=0 \mathrm{~s}, 10$ with 5-10 s prediction, and 14 with $k_{c}=20 \mathrm{~s}$.

Figure 26 shows a summary of the preceding discussion and the decrease in total number of PMA activations when more sophisticated control logic is employed. As seen, it resulted in a

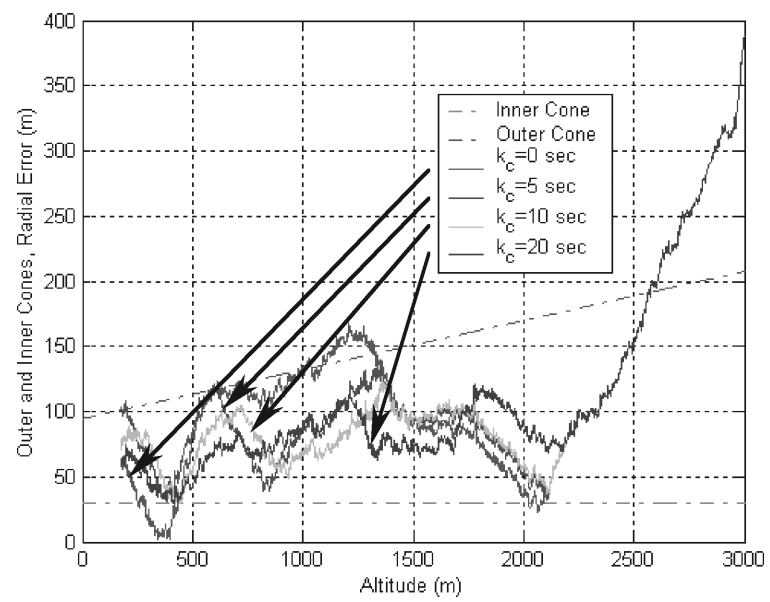

Fig. 25 Introducing a prediction term.

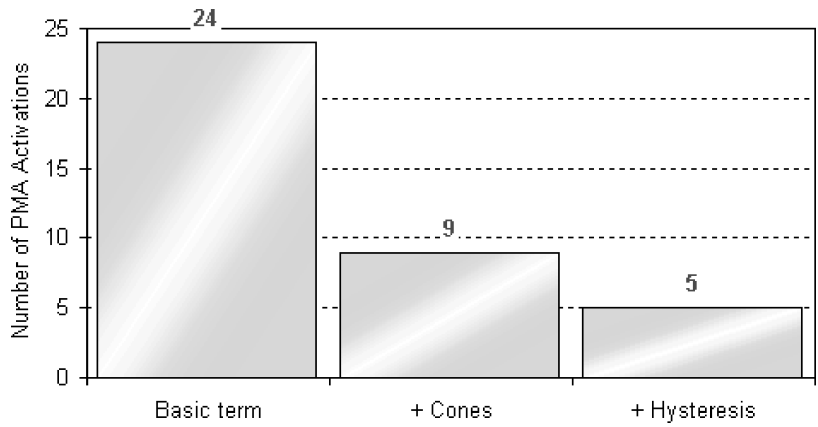

Fig. 26 Number of PMA activations decrease.

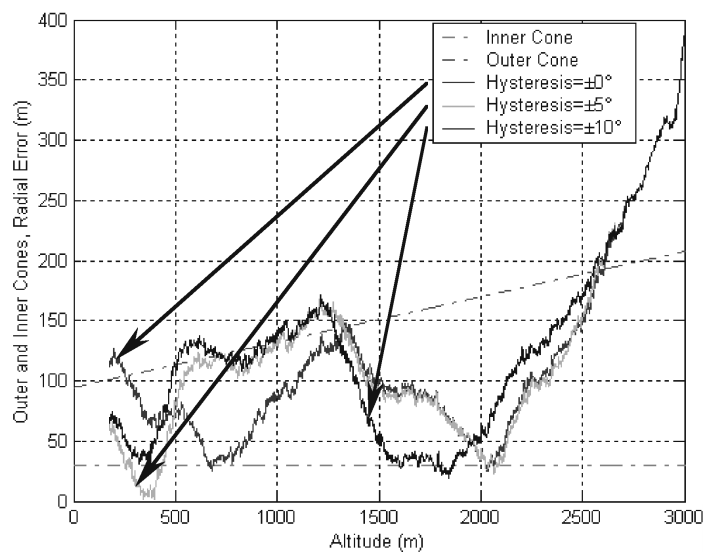

b)

Fig. 24 To fight yaw oscillations, introduction of a) delay and b) hysteresis. 


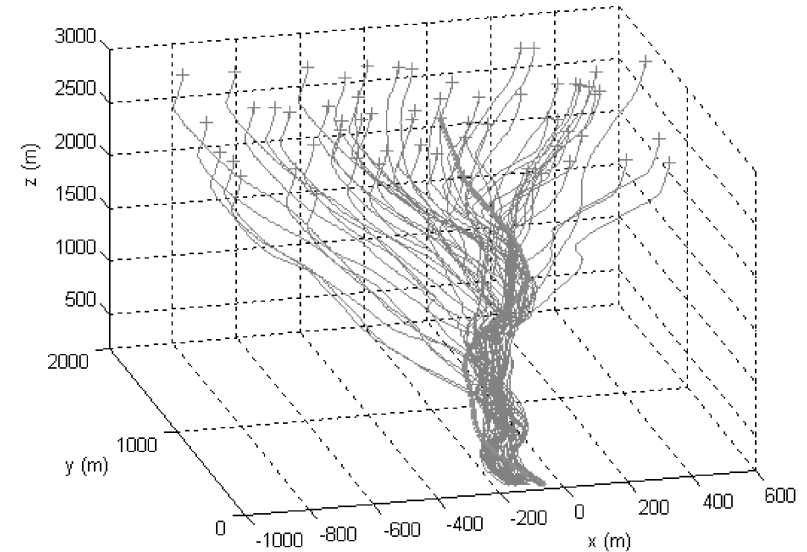

a)

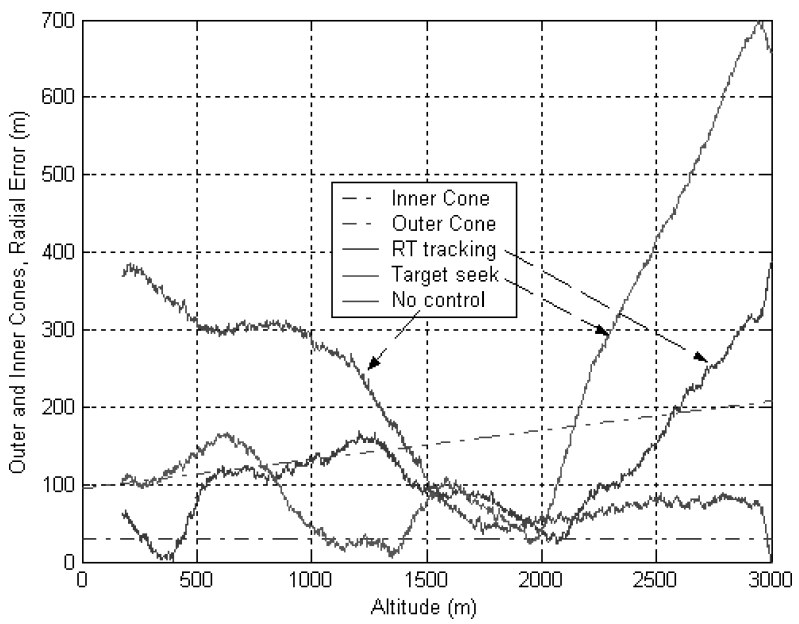

Fig. 28 RT-tracking vs target-seek trajectories.

significant reduction in the number of PMA activations in comparison with the basic classical control logic. This ensured an almost sevenfold increase in the reserve with respect to available control authority (32 activations).

Figure 27 shows an example of Monte Carlo simulation with the same wind profile and two control strategies. The first one employs basic terms, cones, and 10-s delay. The second one uses basic terms, 5-deg hysteresis instead of delay, and prediction term (24) with $k_{c}=25 \mathrm{~s}$. The thick central curves on both graphs represent the RT. When Fig. 27 is observed and the results of the simulations are analyzed, it can be stated that the second algorithm performs much better. Not only is the mathematical expectation of the final position error for the second algorithm twice as small as for the first one, but the standard deviation decreases by a factor of three. Whereas replacing the 10-s delay with a 5-deg hysteresis leads to the fourfold decrease of the number of PMA activations without a deterioration of the overall performance, introducing a prediction term costs a twofold increase of PMA activations so that on average the first algorithm required twice as many activations as the second.

Finally, Fig. 28 includes the results of a simulation where $\boldsymbol{P}_{I}$ is defined using Eq. (27), also known as a target-seek control strategy. These results are compared with the standard RT-tracking strategy (17). All inputs are the same for both simulations including $\Delta=143 \mathrm{deg}$, solid-edge cones (around the RT in the first case and around vertical line stretching upward from the target), and \pm 5 -deg hysteresis. Both algorithms perform well, requiring only five PMA activations each.

Figure 28 also includes the simulation run where no control was used to steer the parachute. Even when being released exactly at the CARP (with no initial error), the uncontrolled parachute flies away from the RT ending up with almost 400-m error at the touchdown

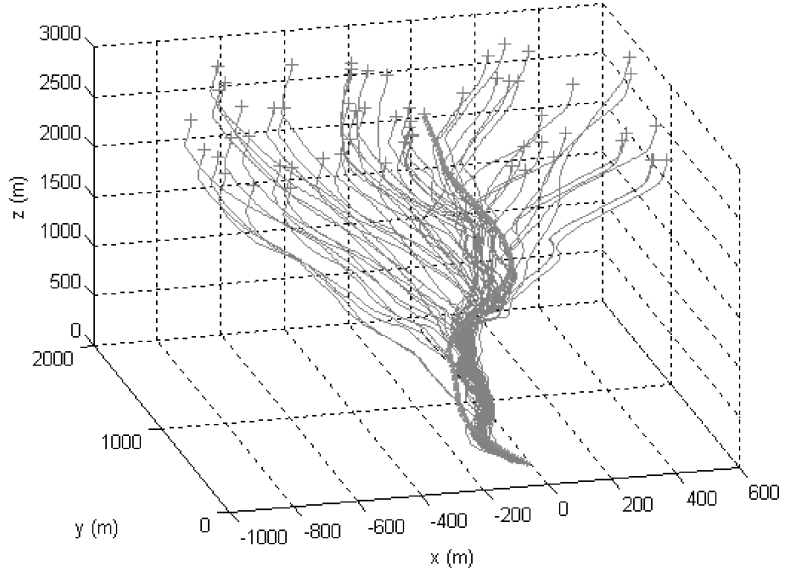

b)

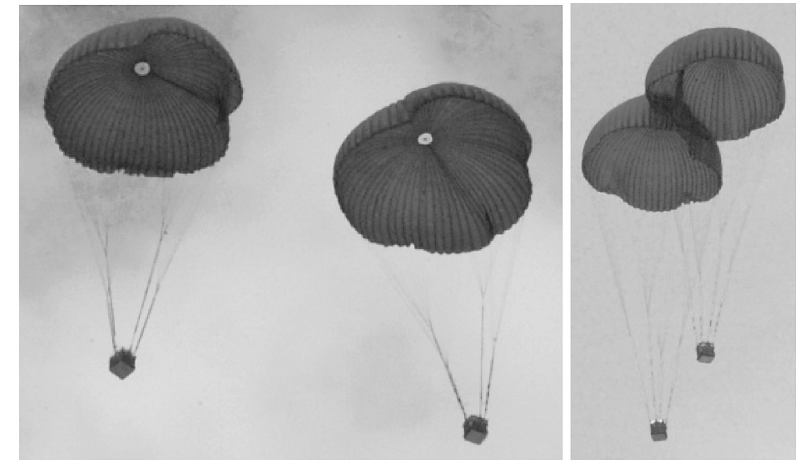

Fig. 29 Two AGAS steering toward the same RT.

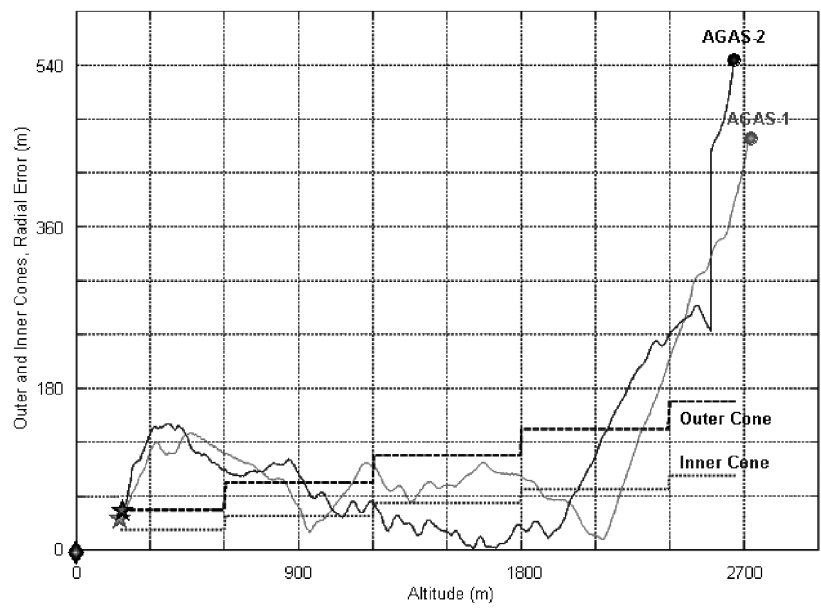

Fig. 30 Drops, 13 September, $3000 \mathrm{~m}$

point. Clearly, the reason for this is that the predicted wind profile was not sufficiently accurate. However, the controlled parachute with a reasonable algorithm suggested by Eq. (26) handles this situation fairly well.

\section{Flight Test}

A total of about 15 controlled drops were made at YPG to test the AGAS concept and control algorithm. The final demonstration took place at YPG during the Precision Airdrop Technology Conference and Demonstration (PATCAD) on 13 and 14 September 2001.**

\footnotetext{
${ }^{* *}$ Information about the PATCAD can be found online at the following YPG-sponsored web site: http://yuma-notes1.army.mil/mtea/patcadreg.nsf [cited September 2001].
} 
During preliminary tests, a ground station was used to control AGAS via a wireless modem. ${ }^{18}$ The AGAS sent its current position and yaw angle to the ground station. The ground station processed the data using the flight control algorithm and then issued appropriate commands to the AGAS GNC. For the final drops, all GNC algorithms were executed aboard AGAS. The downlink message was used for real-time monitoring during the drop.

The rest of the section summarizes the results of two successful drops of four AGAS performed during the final PATCAD demonstration (Table 1). (On 14 September AGAS-3 quit working half the way down because of a valve system malfunction.) The miss distance for three operable AGAS systems released was less than $78 \mathrm{~m}$ as oppose to $140-1370 \mathrm{~m}$ for uncontrolled parachutes.

Table 1 PATCAD results

\begin{tabular}{lcc}
\hline \hline Test item & Weight, kg & IP miss, m \\
\hline & 13 & September 2002 \\
WindPack & 21 & 515.1 \\
Standard G-12 & 724 & 512.2 \\
Standard G-12 & 773 & 141.9 \\
AGAS-1 & 726 & 76 \\
AGAS-2 & 726 & 78 \\
& 14 September 2002 \\
WindPack & 21 & 1048.6 \\
Standard G-12 & 726 & 1371.6 \\
AGAS-3 & 726 & 347.3 \\
AGAS-4 & 726 & 55.5 \\
\hline \hline
\end{tabular}

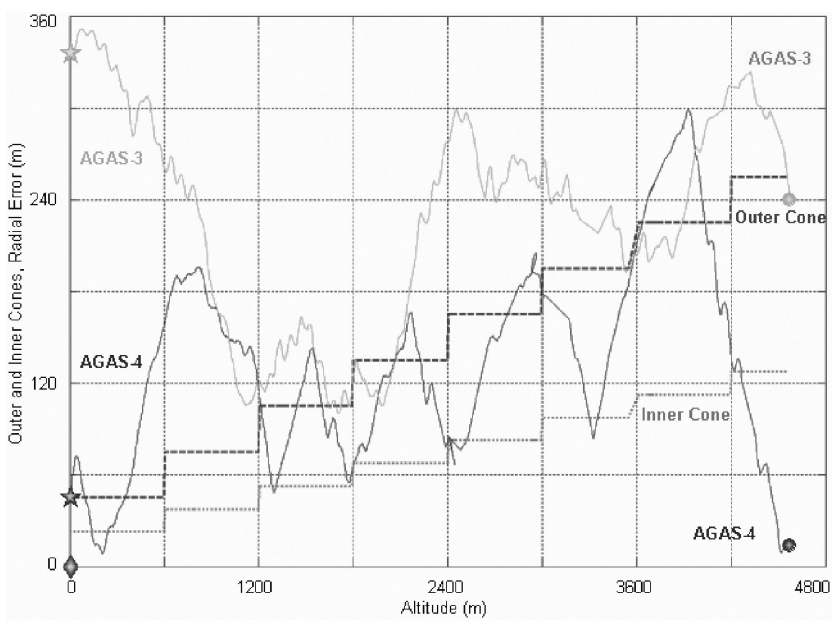

Fig. 31 Drops, 14 September, $4500 \mathrm{~m}$.
The same control algorithm employed on AGAS-1 and AGAS-2 led to impact of the two systems during the first drop, as shown in Fig. 29. For the second drop, different target areas were input into the GNC systems of the two parachutes to avoid possible collision.

Figure 30 shows the integral data for two first-day drops from the altitude of $3000 \mathrm{~m}$. The 30-min old wind data was used to compute the RT. Despite a large initial error, both AGAS steered to the TA fairly well: 17 and 18 PMA activations were needed to hit the target, with approximately the same miss distance.

Figure 31 presents the same set of data for the second drop of the AGAS on 14 September (released at $4500 \mathrm{~m}$ ). The wind profile used for this drop was $2 \mathrm{~h}$ old. Observe that AGAS-4 drifts away from the CARP due to a bad wind estimate for the first $1000 \mathrm{~m}$. However, on leaving the outer cone, it is steered back inside. As soon as the PMAs inflate on entering the inner cone, the AGAS proceeds to drift out again.

Figure 32 shows the control-related data for the AGAS-4; 28 PMA fills were needed to hit the target with a 55-m miss.

\section{Conclusions}

This paper addressed the development of a GNC system for a circular parachute. The design methodology presented was based on the optimal control and Lyapunov stability analysis of the problem. The optimal control analysis has resulted in the concept of the OA that turned out to be the central element of the GNC strategy. The Lyapunov stability analysis has derived the feasible range of the OAs. As a result, a bang-bang control strategy imposed by the PMA hardware was developed to drive successfully a circular parachute to the TA within the prescribed circular error. Furthermore, to reduce the number of actuations (the requirement driven by the finite amount of nitrogen available onboard) the concepts of inner and outer coned were added. These sufficiently reduced the number of actuations to make the bang-bang GNC strategy feasible, that is, the amount of nitrogen available could support the required number actuations. Further reduction in the number of actuations was achieved by introducing a derivative term in the control strategy, as well as hysterisis in the definition of the OA. The paper includes a thorough simulation analysis of the proposed GNC strategy, as well as flight-tests results obtained at YPG in September of 2001 during PATCAD. All of the results, both from simulation and from flight test, indicate that the proposed GNC strategy achieved and exceeded the design specifications.

\section{Acknowledgments}

The authors thank the U.S. Army Yuma Proving Ground and the U.S. Army Soldier and Biological Chemical Command team members as well as Vertigo, Inc., Charles Stark Draper Laboratory, Inc., and PSI, Inc., team members for their help with the flighttest setup. We also thank all Naval Postgraduate School graduate students who participated in the project.

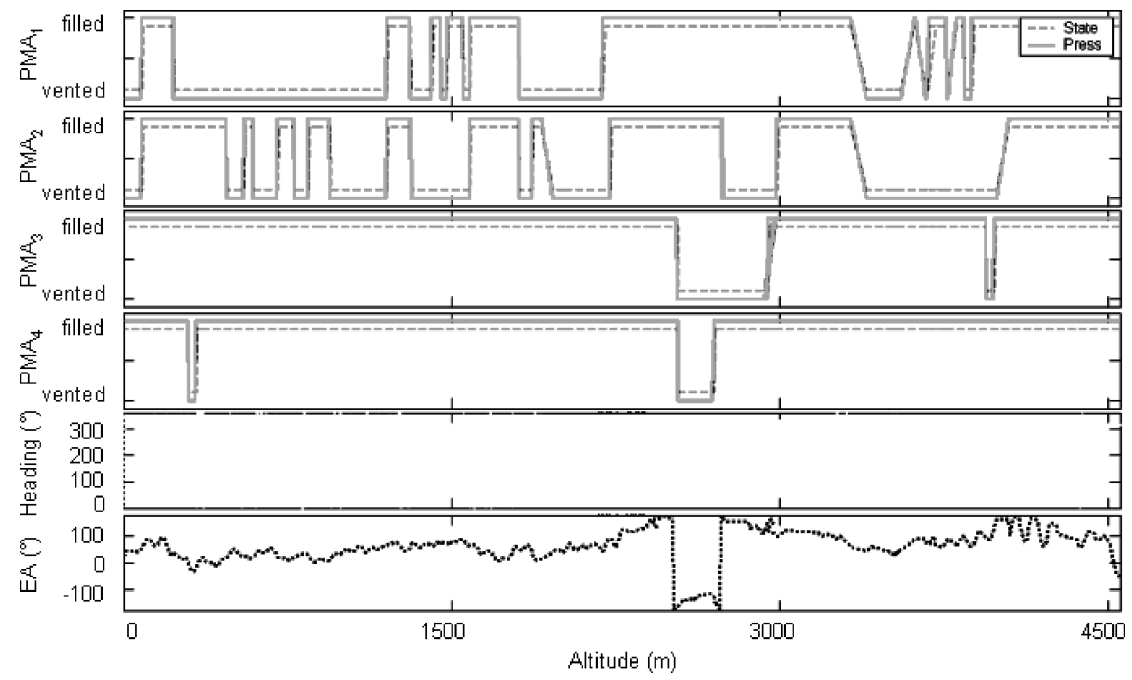

Fig. 32 AGAS-2 control data. 


\section{References}

1"Summary Report: New World Vistas, Air and Space Power for the 21st Century,” U.S. Air Force Science Advisory Board, 1997.

${ }^{2}$ Benney, R., and Accorsi, M., "Aerodynamic Decelerator Systems: Diverse Challenges and Recent Advances," Journal of Aircraft, Vol. 38, No. 5, 2001, pp. 785-785.

${ }^{3}$ Dellicker, S., Benney, R., and Brown, G., "Guidance and Control for FlatCircular Parachutes," Journal of Aircraft, Vol. 38, No. 5, 2001, pp. 809-817.

${ }^{4}$ Dellicker, S., "Low Cost Parachute Navigation Guidance and Control," M.S. Thesis, Dept. of Aeronautics and Astronautics, Naval Postgraduate School, Monterey, CA, Sept. 1999.

${ }^{5}$ Hattis, P., Fill, T., Rubenstein, D., Wright, R., Benney, R., and LeMoine, D., "Status of an On-Board PC-Based Airdrop Planner Demonstration," AIAA Paper 2001-2066, May 2001.

${ }^{6}$ Forehand, J. E., “The Precision Drop Glider (PDG)," U.S. Air Force Rept. FTC-TR-64-12, 1969, pp. 24-44.

${ }^{7}$ Wailes, W., and Harrington, N., "The Guided Parafoil Airborne Delivery System Program,” AIAA Paper 95-1538, May 1995.

${ }^{8}$ Smith, B. D., "Steering Algorithms for GPS Guidance of RAM-AIR Parachutes," Proceedings the 8th International Technical Meeting of the Institute of Navigation, Inst. of Navigation, Fairfax, VA, 1995.

${ }^{9}$ Final Report: Development and Demonstration of a Ram-Air Parafoil Precision Guided Airdrop System, Vol. 3, Charles Stark Draper Lab. Inc., U.S. Army Contract DAAK60-94-C-0041, Oct. 1996.

${ }^{10}$ Williams, T., "Optimal Parachute Guidance, Navigation, and Control for the Affordable Guided Airdrop System (AGAS)," M.S. Thesis, Dept. of Aeronautics and Astronautics, Naval Postgraduate School, Monterey, CA, June 2000

${ }^{11}$ Dellicker, S., Benney, R., Patel, S., Williams, T., Hewgley, C., Yakimenko, O., Howard, R., and Kaminer, I., "Performance, Control and Simulation of the Affordable Guided Airdrop System," AIAA Paper 20004309, Aug. 2000.

${ }^{12}$ Dobrokhodov, V., Yakimenko, O., and Junge, C., "Six-Degree-ofFreedom Model of a Controlled Circular Parachute," Journal of Aircraft, Vol. 40, No. 3, 2003, pp. 482-493.

${ }^{13}$ Yakimenko, O., Dobrokhodov, V., Johnson, J., Kaminer, I., Dellicker, S., and Benney, R., "On Control of Autonomous Circular Parachute," AIAA Paper 2002-4753, Aug. 2002.

${ }^{14}$ Knacke, T. W., Parachute Recovery Systems Design Manual, Para, Santa Barbara, CA, 1992.

${ }^{15}$ Benney, R., Brown, G., and Stein, K., “A New Pneumatic PMA: Its Use in Airdrop Applications," AIAA Paper 99-1719, June 1999.

${ }^{16}$ Kelly, K., and Pena, B., "Wind Study and GPS Dropsonde Applicability to Airdrop Testing," AIAA Paper 2001-2022, May 2001.

${ }^{17}$ Pontrjagin, L., Boltjanskiy, V., Gamkrelidze, R., and Mishenko, E., Mathematical Theory of Optimal Processes, Nayka, Moscow, 1969 (in Russian).

${ }^{18}$ Johnson, J., Yakimenko, O., Kaminer, I., and Dellicker, S., "On the Development and Pre-Flight Testing of the Affordable Guided Airdrop System for G-12 Cargo Parachute,’ AIAA Paper 2001-2060, May 2001.

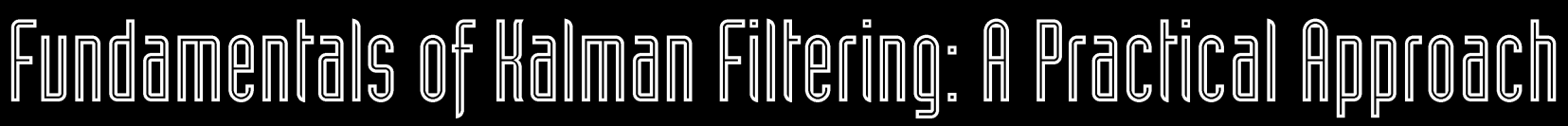

Paul Zarchan and Howard Musoff, C.S. Draper Laboratory

$\overline{7}^{\mathrm{h}}$ his text is a practical guide to building Kalman filters and shows how the filtering equations can be applied to real-life problems. Numerous examples are presented in detail showing the many ways in which Kalman filters can be designed. Computer code written in FORTRAN, MATLAB, and True BASIC accompanies all of the examples so that the interested reader can verify concepts and explore issues beyond the scope of the text.

Sometimes mistakes are introduced intentionally to the initial filter designs to show the reader what happens when the filter is not working

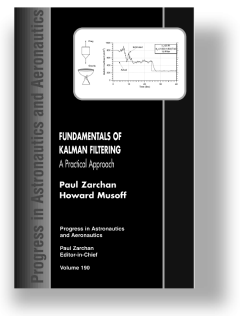

properly. The text spends a great deal of time setting up a problem before the Kalman filter is actually formulated to give the reader an intuitive feel for the problem being addressed. Real problems are seldom presented in the form of differential equations and they usually don't have unique solutions.
Therefore, the authors illustrate several different filtering approaches for tackling a problem. Readers will gain experience in software and performance tradeoffs for determining the best filtering approach for the application at hand.

Progress in Astronautics and Aeronautics 2000, 670 pp, Hardcover

ISBN 1-56347-455-7

List Price: $\$ 99.95$

AIAA Member Price: $\$ 74.95$

Source: 945

\section{MIAa}

American Institute of Aeronautics and Astronautics
American Institute of Aeronautics and Astronautics

Publications Customer Service, P. B Box 960. Herndon. VA 20172-0960

Fax: 703/661-1501 • Phone: 800/682-2422 • E-mail: warehouse@aiaa.org

Order 24 hours a day at www.aiaa.org 